\title{
Design and experimental study of a C-band traveling-wave accelerating structure
}

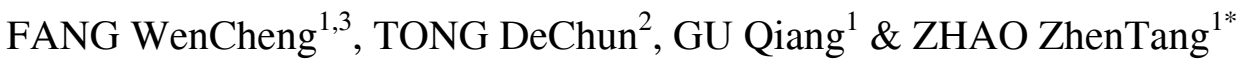 \\ ${ }^{1}$ Shanghai Institute of Applied Physics (SINAP), Chinese Academy of Sciences, Shanghai 201800, China; \\ ${ }^{2}$ Department of Engineering Physics, Tsinghua University, Beijing 100084, China; \\ ${ }^{3}$ Graduate University of Chinese Academy of Sciences, Beijing 100039, China
}

Received June 29, 2010; accepted August 20, 2010

\begin{abstract}
Research and design of a C-band (5712 MHz) high-gradient traveling-wave accelerating structure is being carried out at Shanghai Institute of Applied Physics, Chinese Academy of Sciences. The structure consists of 53 regular disk-loaded cells and two waveguide couplers, and its length is about $1 \mathrm{~m}$. This paper presents a design method for the accelerating structure, an experimental model and the preliminary results of an RF cold test of the model structure.
\end{abstract}

C-band, high gradient, constant gradient, waveguide coupler, tuning

Citation: Fang W C, Tong D C, Gu Q, et al. Design and experimental study of a C-band traveling-wave accelerating structure. Chinese Sci Bull, 2011, 56: 18-23, doi: 10.1007/s11434-010-4265-2

Shanghai Synchrotron Radiation Facility (SSRF), a thirdgeneration light source in China, commenced routine user operation in May 2009 [1]. As a further development of light sources, a compact hard X-ray free electron lasers (FEL) facility is presently being planned at Shanghai Institute of Applied Physics (SINAP), Chinese Academy of Sciences. This facility will be located close to SSRF and thus requires a compact linac with a high-gradient accelerating structure for a limited overall length of less than 650 m. A room-temperature linac operated at higher frequency with smaller scale can provide a higher accelerating gradient. However, a smaller-scale structure induces a serious wakefield and high gradient results in breakdown of the electric field. The C-band (5712 MHz) accelerating structure described in this paper is a good compromise for this compact facility. The technology of C-band accelerating structures has been well established at KEK and RIKEN/ SPring-8. Field gradients have reached $45 \mathrm{MV} / \mathrm{m}$ at KEK [2] and $40 \mathrm{MV} / \mathrm{m}$ at SPring-8 [3].

The C-band accelerating structure under development at SINAP has a constant acceleration gradient, and it is planned

*Corresponding author (email: zhaozt@ sinap.ac.cn) for the operational gradient to exceed $40 \mathrm{MV} / \mathrm{m}$, which is about twice that of the $\mathrm{S}$ band. A waveguide coupler, which is an electric coupler with two coupling ports, is used to input power. Comparing with a single-port magnetic coupler, the waveguide coupler has the advantages of a lower rate of breakdown and elimination of the asymmetry dipole field in the coupler cavity for high beam quality [4,5]. This paper is a design study of the constant gradient structure and waveguide coupler. The results of a radio frequency (RF) cold test of the experimental model are found to be in good agreement with design values.

\section{Method employed in designing the traveling- wave accelerating structure}

A traveling-wave accelerating structure comprises a regular section and input and output couplers. Cells of the regular section should be distributed in a specified sequence and satisfy the constant gradient condition. The input and output couplers should match the regular section and rectangular waveguides to input and output RF power with no reflection 
from either the input or output coupler.

\subsection{Constant-gradient structure}

According to the theory of a constant-gradient structure [6], the group velocity of each cell varies linearly as

$$
v_{\mathrm{g}}=\frac{\omega L}{Q}\left\{\left[1-\left(1-\mathrm{e}^{-2 \tau}\right) z / L\right] /\left(1-\mathrm{e}^{-2 \tau}\right)\right\},
$$

where $\omega$ is the operating frequency, $L$ is the effective length, $\tau$ is the attenuation factor of the accelerating structure, and $z$ is the longitudinal coordinate on the axis. Under the condition of a flat-top input power pulse, the electric field amplitude on the axis is constant periodically.

\subsection{Design of the traveling-wave cell using SUPER- FISH}

The computer software SUPERFISH [7] can calculate the parameters of the traveling-wave (TW) structure as well as those of a standing-wave (SW) structure [8]. In SUPERFISH, shunt impedance $R$, stored energy $W$ and resonant frequency $f$ are provided for the SW directly. $R$ and $f$ are also available for the TW; however, $W_{\mathrm{TW}}=W_{\mathrm{SW}} / 2$, and the group velocity for the $\mathrm{TM}_{01}$ mode of the disk-loaded structure is

$$
v_{\mathrm{g}}=\frac{P}{W_{\mathrm{TW}}}=\frac{\frac{1}{2} \int E_{r} \cdot H_{\phi} \mathrm{d} S}{\int_{\substack{\text { unit } \\ \text { length }}} \frac{\varepsilon E^{2}}{2} \mathrm{~d} V+\int_{\substack{\text { unit } \\ \text { length }}} \frac{\mu H^{2}}{2} \mathrm{~d} V},
$$

where $W_{\mathrm{TW}}=W_{\mathrm{SW}} / 2$ and $P$ derived from the Poynting vector is the power penetrating through the surface, which is perpendicular to the direction of the group velocity. $E_{r}$ and $H_{\phi}$ are transversal components of the TW field. Therefore, the next step is to derive $E_{r}$ and $H_{\phi}$ for the TW from the SW field.

In the SUPERFISH model shown in Figure 1, transversal components of the TW can be derived from the $\mathrm{SW}$ in the $2 \pi / 3$ dispersion mode as expressed by eq. (3) under the Newman boundary condition $\left(E_{r}=0\right)[8]$ :

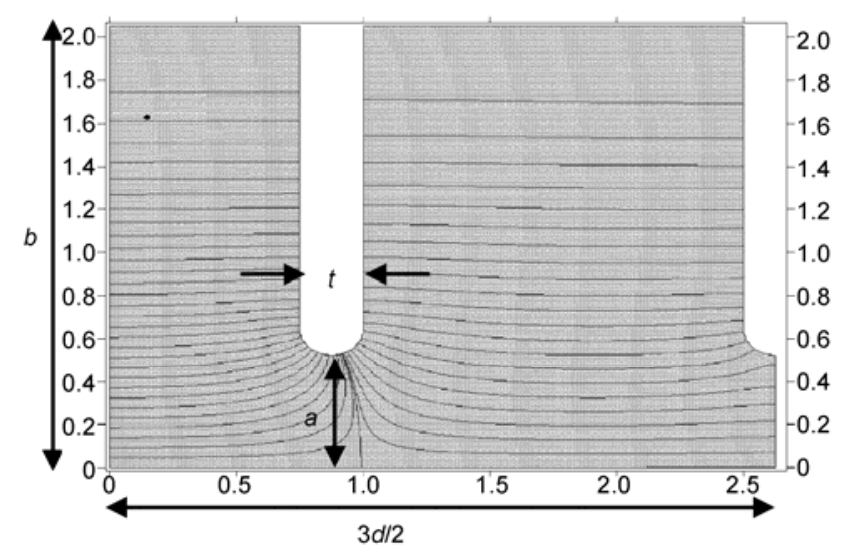

Figure 1 SUPERFISH model for TW cell calculation. This model comprises 1.5 cells.

$$
\begin{aligned}
& \operatorname{Disk}\left(\frac{d}{2}\right): E_{r, \mathrm{TW}}=E_{r, \mathrm{SW}}\left(\frac{d}{2}\right) / \sqrt{3}, H_{\phi, \mathrm{TW}}=H_{\phi, \mathrm{SW}}(d), \\
& \operatorname{Mid}-\operatorname{cavity}(d): E_{r, \mathrm{TW}}=E_{r, \mathrm{SW}}(d) / \sqrt{3}, H_{\phi, \mathrm{TW}}=H_{\phi, \mathrm{SW}}(d) .
\end{aligned}
$$

The TW structure parameters can then be calculated cell by cell.

\subsection{Method of coupler design}

Couplers should match the regular section and rectangular waveguides. For this design, a waveguide coupler with two ports is adopted for the accelerating structure, and Kroll's method based on the time domain is employed to tune the coupler [9]. This method satisfies both external (conventional) and internal matching conditions (both the amplitude and phase of the accelerating field on the axis are observed), and thus it eliminates the possibility that the match arises from a fortuitous cancellation between forward and backward waves within the accelerator structure. It is assumed that the electric field amplitude on the axis is

$$
E_{c}(z)=E(z)(\exp (-j \phi(z))+R \exp (j \phi(z))),
$$

where $E(z)$ is a real positive amplitude function, $\phi(z)$ is a real phase function, and $R$ is the reflection coefficient of the output coupler independent of $z$. From Floquet's theorem,

$$
E(z \pm P)=E(z), \text { and } \phi(z \pm P)=\phi(z) \pm \psi,
$$

where $P$, which is the length of a cell in this design, is the period of the accelerating structure and $\Psi$ is the phase advance per cell. Thus,

$$
\begin{gathered}
2 \cos (\psi)=\sum(z), \\
R \exp (2 j \phi)=[2 \sin (\psi)-j \Delta(z)] /[2 \sin (\psi)+j \Delta(z)],
\end{gathered}
$$

where

$$
\begin{aligned}
& \sum(z)=F^{+}(z)+F^{-}(z), \\
& \Delta(z)=F^{+}(z)-F^{-}(z), \\
& F^{ \pm}(z)=E_{c}(z \pm P) / E_{c}(z) .
\end{aligned}
$$

Discrete $E_{c}(z)$ data can be exported from the simulation software. Taking Microwave Studio-CST (MWS) for example, the scale of couplers can be adjusted iteratively according to the data analysis. Tuning is accomplished when $|R|=0$ and $\Psi$ is equal to the phase advance per cell.

\section{Design optimization and simulation}

The present C-band accelerating structure comprises 53 regular cells and input and output waveguide couplers, and the total length is about $1 \mathrm{~m}$, as shown in Figure 2. The gradient of the field should be constant. The operating frequency is $5712 \mathrm{MHz}$ and the operating mode is $2 \pi / 3$. 

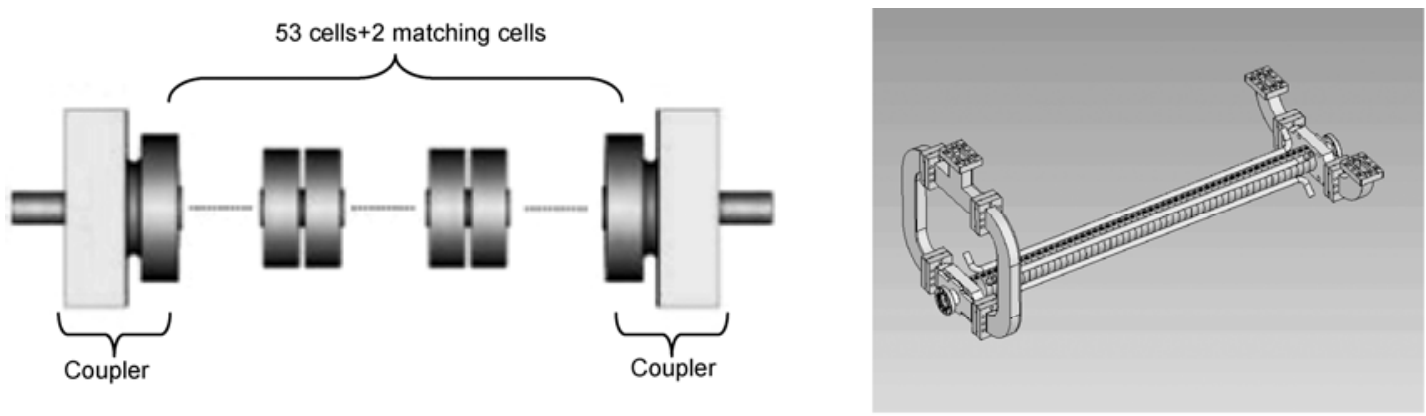

Figure 2 Overall accelerating structure.

\subsection{Regular section design using SUPERFISH}

FISH of SUPERFISH is adopted for calculating the 53 regular cells [8]. The model illustrated in Figure 1 is used to calculate the properties of cells including the scale and resonant frequency, shunt impedance, $Q$ value and group velocity. Three parameters can be adjusted for tuning: cell length $d$, cell diameter $2 b$ and iris aperture diameter $2 a . d$ is determined by the $5712 \mathrm{MHz}$ resonant frequency and the phase velocity equals the light velocity; therefore, only two adjustable parameters remain. According to the simulation, the resonant frequency shift is dominated by $2 b$ about 150 $\mathrm{kHz} / \mathrm{mm}$, and the group velocity is dominated by $2 a$.

$2 a$ and $2 b$ are tuned iteratively to reach the target resonant frequency and group velocity. The electric field amplitude on the axis after iterative calculations for the 53 cells is illustrated in Figure 3, and the characteristic parameters of the TW structure are presented in Table 1.

\subsection{Coupler design using MWS of CST}

MWS software is used for three-dimensional electromag-

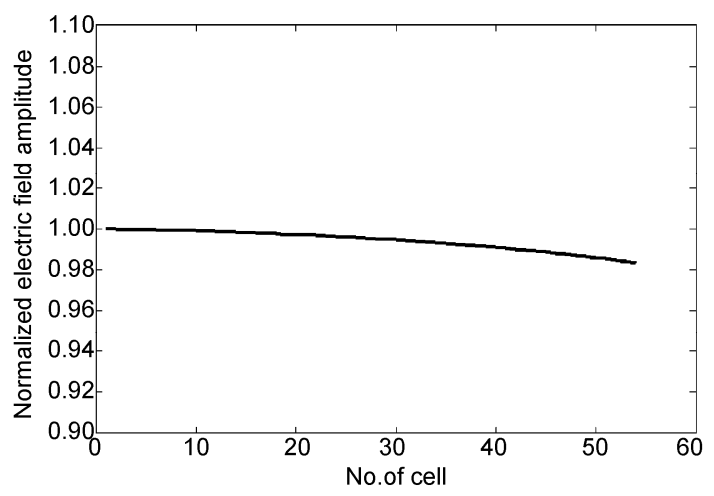

Figure 3 Field amplitude distribution on the axis. Each data point represents the middle point of a cell.

netic simulation. Matlab, with powerful numerical capability, is used as a postprocessor employing eq. (6). VBA (Visual Basic Application) of CST is used as a coordinator for MWS-Matlab parallel work.

Considering the running time and limited memory of the computer, a novel MWS model was designed for simulation, as shown in Figure 4. The model is made of two identical

Table 1 Parameters of the regular section

\begin{tabular}{ccccccc}
\hline Operating frequency $f$ & Number of cells & Operating mode & Length of structure & Length of cell $d$ & Disk thickness $t$ & Diameter of aperture $2 a$ \\
\hline $5712.000 \mathrm{MHz}$ & 53 & $2 \pi / 3$ & $944.73 \mathrm{~mm}$ & $17.495 \mathrm{~mm}$ & $2.500 \mathrm{~mm}$ & $10.405-12.475 \mathrm{~mm}$ \\
\hline \hline Diameter of cell $2 b$ & Shunt impendence $R_{\mathrm{s}}$ & Quality factor $Q$ & Group velocity $v_{\mathrm{g}} / c$ & Filling time $t_{\mathrm{F}}$ & Attenuation factor $\tau$ & \\
\hline $41.001-41.492 \mathrm{~mm}$ & $76.19-87.18 \mathrm{M} \Omega / \mathrm{m}$ & $9893-9864$ & $1.88 \%-1.00 \%$ & $226 \mathrm{~ns}$ & 0.4565 & \\
\hline
\end{tabular}
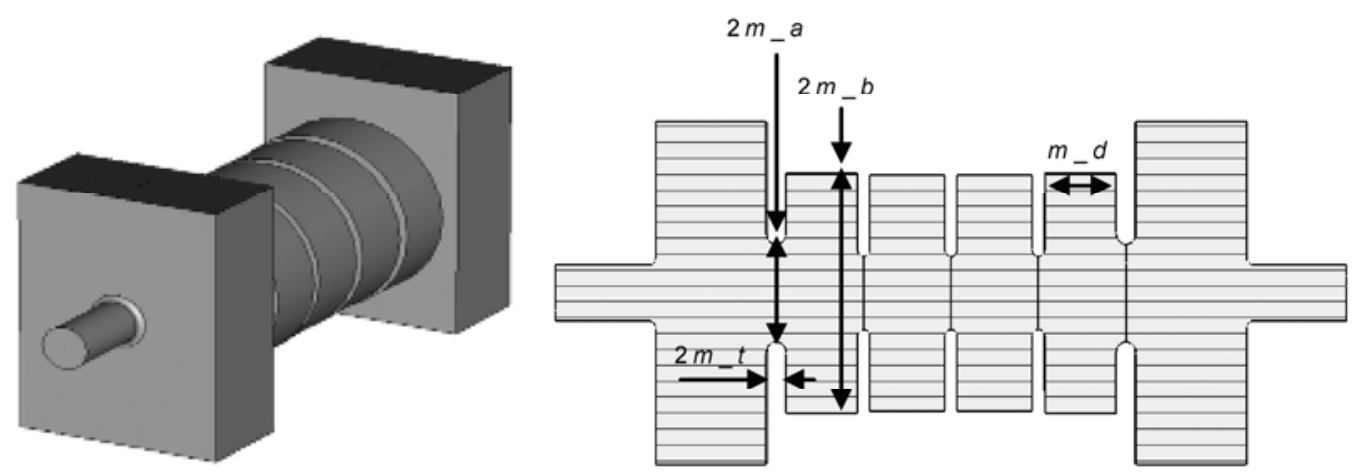

Figure 4 Three-dimensional model for MWS simulation. 
regular cells and two identical couplers. A coupler includes a rectangular waveguide and a matching cell. The scale of regular cells is fixed by the SUPERFISH simulation, and only the scale of the two couplers is adjustable in MWS.

There are four parameters of the matched cell for tuning the coupler, as shown in Figure 4. However, only $2 m \_a$ and $2 m \_b$ are tuned, and $2 m \_t$ and $m \_d$ are fixed.

$2 m \_a$ and $2 m \_b$ are tuned for the coupling coefficient and frequency respectively. Both are tuned iteratively when the coupler is matched. The optimum parameters are 24.474 and $44.924 \mathrm{~mm}$ for $2 m \_a$ and $2 m \_b$, respectively, and $2 m \_t$ and $m \_d$ are fixed at 3 and $17.495 \mathrm{~mm}$, respectively.

Figures 5 and 6 present the simulation results. In Figure $5,|R|$ of the mismatched coupler is very large. In the matched case, the real part of $\cos \Psi$ is -0.5 and the imaginary part is zero, and $|R|$ is 0.0135 between the two vertical red dashed lines, which is the effective area for eq. (6) [9]. All results demonstrate that the couplers are matched and the phase advance is $120^{\circ}$, which is the design value. Figure 6 illustrates the field and phase distribution on the axis. The field in the coupler is lower than that in the regular section, and the phase advance between the middle points of the two matching cells is about $360^{\circ}$. Figure 6 shows the phenomenon that the phase velocity of the rectangular waveguide is about $0.6 c$, which is lower than the speed of a relativistic electron, and the beam dynamics of electrons with low velocity may be complicated because of non-synchronization between a bunch and traveling-wave; however, the field in the waveguide is very weak, and the transversal and longitudinal fields imposed on the beam can be omitted.

The above provides a systematic method for the design of a TW accelerating structure. The regular section can be designed cell by cell with SUPERFISH, and the input and output couplers can be calculated by MWS. However, there may be some slight mismatch between the simulation model and experimental model, which can be tuned in an RF cold test step as discussed in the next section.

\section{Structure fabrication and RF measurements}

To check the structure design, an experimental model
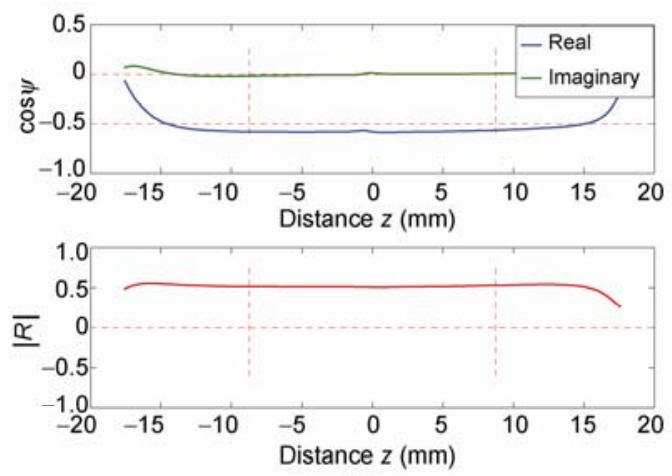

(a)

Figure $5 \Psi$ and $R$ for mismatched (a) and matched (b) couplers. including 12 regular cells and two couplers was fabricated. The model is composed of an input coupler, output coupler and 12 identical regular cells (No. 53 cell of the constant structure in Section 2.1), as shown in Figure 7. The $Q$ value, frequency and field distribution on the axis were measured, and the results were also acquired in tuning experiments.

\subsection{Frequency and $Q$ value measurement}

The design frequency of the $2 \pi / 3$ mode was $5712 \mathrm{MHz}$. However, other modes were also investigated. Five cells were measured and the results are presented in Table 2. In this table, the coupling coefficient $k$ is derived from the frequency of three modes, and there is about $3.5 \mathrm{MHz}$ shift between the RF test and simulation for $2 \pi / 3$. However, the tolerance is about $\pm 300 \mathrm{kHz}$, which indicates stability.

\subsection{Tuning experiment}

As introduced in Section 3.1, there is a resonant frequency shift between the RF cold test and simulation. Four tuning holes are machined on the wall of each cell for frequency tuning. Figure 8 shows the result of tuning. Curve fitting of the measurement data gives a tuning value of about 8 $\mathrm{MHz} / \mathrm{mm}$ for each hole in the linear part. A value of 8 $\mathrm{MHz} / \mathrm{mm}$ for tuning cells is large relative to the $4 \mathrm{MHz}$ residual frequency, and thus two tuning holes for each cell are sufficient.

\subsection{Cold test of the experimental model}

The whole model shown in Figure 7 is the experimental model of the TW accelerating structure. According to non-resonant perturbation theory $[10,11]$, the tuning code was written based on LABVIEW 8.5. The software controls the RF cold test system comprising an NWA Agilent 8362B, step motor and computer, and then acquires the measurement data from the system. The amplitude and phase of the field distribution on the axis are analyzed, and then the residual tuning for each cell can be calculated cell
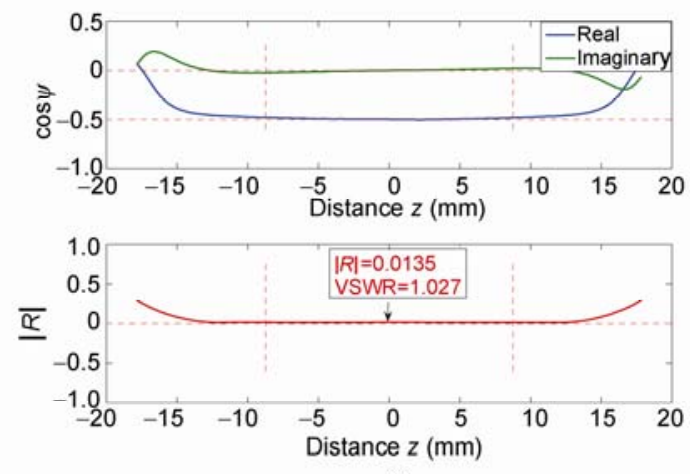

(b) 


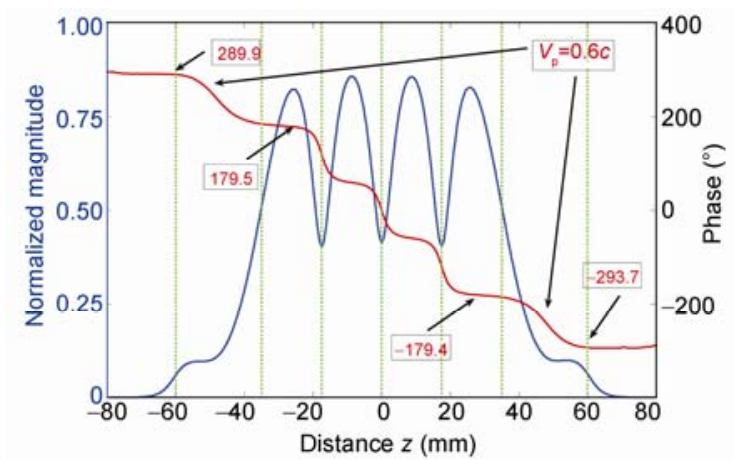

Figure 6 Field and phase distribution for matched couplers.

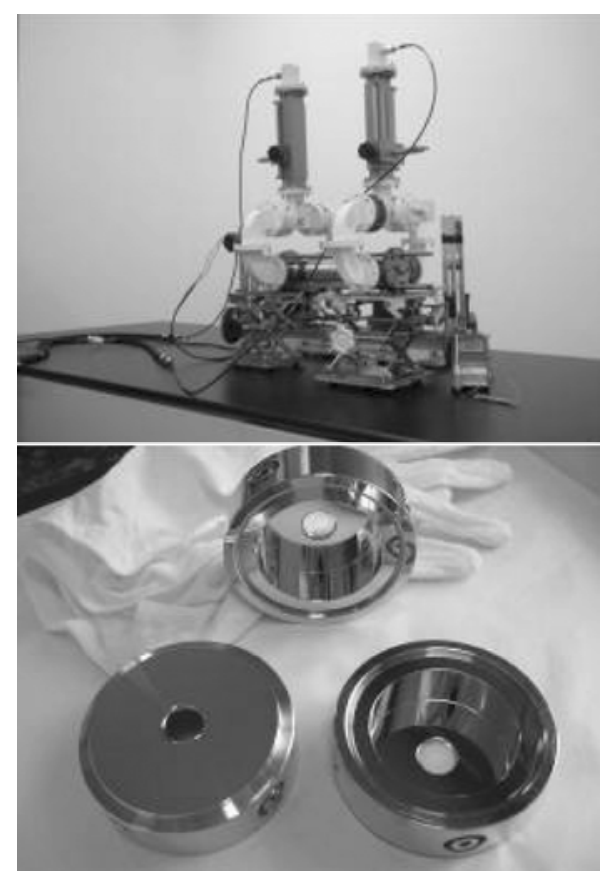

Figure 7 Experimental model and regular cells.

Table 2 Regular-section cell frequency and $Q$ measurement

\begin{tabular}{|c|c|c|c|c|c|}
\hline \multirow{2}{*}{ Cell No. } & \multicolumn{3}{|c|}{ Frequency $(\mathrm{MHz})$} & \multirow{2}{*}{ Coupling coefficient $k(\%)$} & \multirow{2}{*}{$Q$} \\
\hline & $\pi / 3$ & $\pi / 2$ & $2 \pi / 3$ & & \\
\hline A1 & - & 5694.57 & - & - & 9047 \\
\hline A2 & 5678.72 & 5693.67 & 5708.92 & 1.06 & 9498 \\
\hline A3 & 5678.05 & 5693.62 & 5708.67 & 1.08 & 9550 \\
\hline A4 & 5678.3 & 5693.82 & 5708.52 & 1.08 & 9133 \\
\hline A5 & 5678.42 & 5694.27 & 5708.90 & 1.06 & 8850 \\
\hline Ideal & 5680.52 & 5696.26 & 5712 & 1.10 & 9870 \\
\hline
\end{tabular}

by cell. According to the residual tuning value for each cell, the whole structure can be tuned cell by cell iteratively under the control of LABVIEW code. The tuning results are shown in Figures 9-11, and the standing-wave ratio (SWR) is adopted to describe the matching status. In Figures 9-11, 12 regular cells are divided into four periods, and in each period with three cells, the SWR is defined as the largest
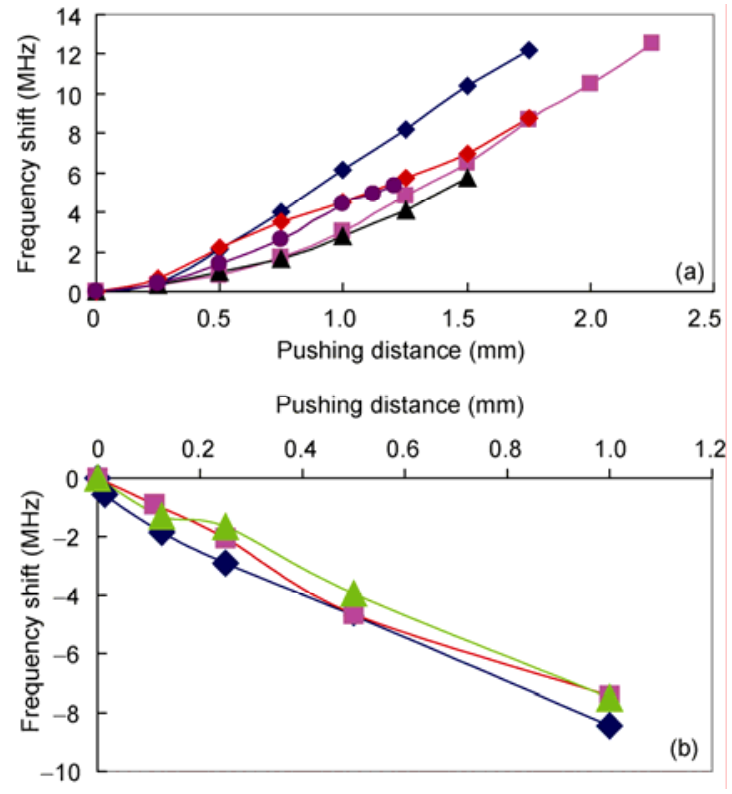

Figure 8 Tuning experiment results. Each curve corresponds to one tuning hole. (a) Pushing the hole (increasing frequency); (b) pulling the hole (decreasing frequency).

field peak divided by the smallest field peak.

There are 14 cells including 12 regular cells in the middle and two matching cells close to the ends. The field distribution in the 12 identical regular cells indicates the matching status of the structure. In Figure 9, the original field distribution corresponding to the design scale is measured before tuning, and there are four quasi-periods corresponding to the middle 12 cells. It is demonstrated that the standing wave comprises two parts, corresponding to the regular section and output coupler, and therefore both the regular section and couplers should be tuned.

First, two single-stub tuners are used to tune the output and input coupler. The field distribution is shown in Figure 10. In this figure, the SWR for the downstream regular cells is lower than that for the upstream regular cells, and therefore there is a reflected wave due to the regular cells. Second, using the LABVIEW code, the regular section is tuned cell by cell and the output coupler is also tuned. The final field distribution is shown in Figure 11. In this figure, the field is distributed periodically and the SWR is about 1.1 . The SWR of the field attenuates from downstream to upstream, indicating that the reflected wave is mainly from the output coupler. In practice, a SWR of about 1.1 is sufficient for high-power conditioning and beam accelerating by the structure.

In Figure 11, the profile of the field distribution is consistent with the simulation result in Figure 6, and the electrical field and phase distribution are nearly identical to the simulation result in Figure 6. There are 14 field peaks in Figure 11 corresponding to the 14 cells in Figure 7, which is more than the number in Figure 6. A greater number of regular cells do not induce more reflection in the structure because regular cells have identical characteristic impedance. 


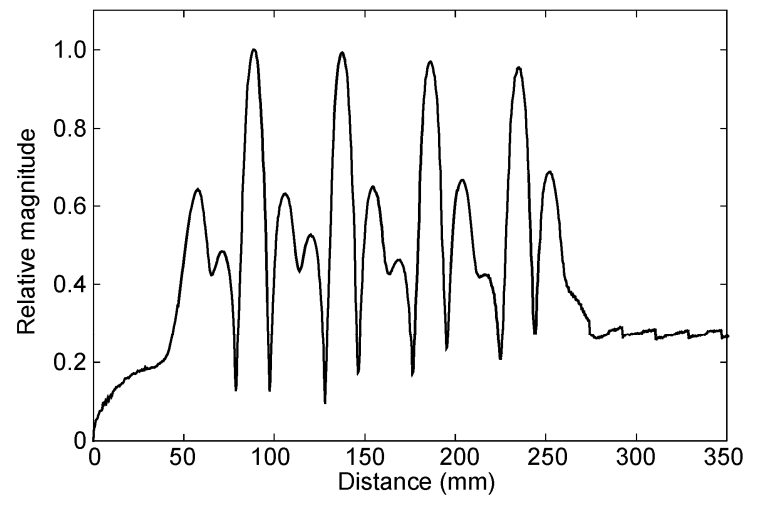

Figure 9 Field distribution before tuning $(\mathrm{SWR}>2)$.

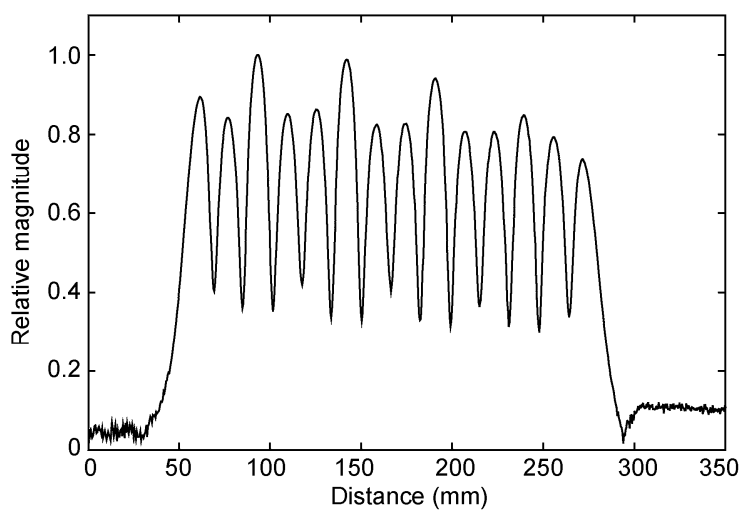

Figure 10 Field distribution after coupler tuning ( $\mathrm{SWR}=1.05-1.2)$.

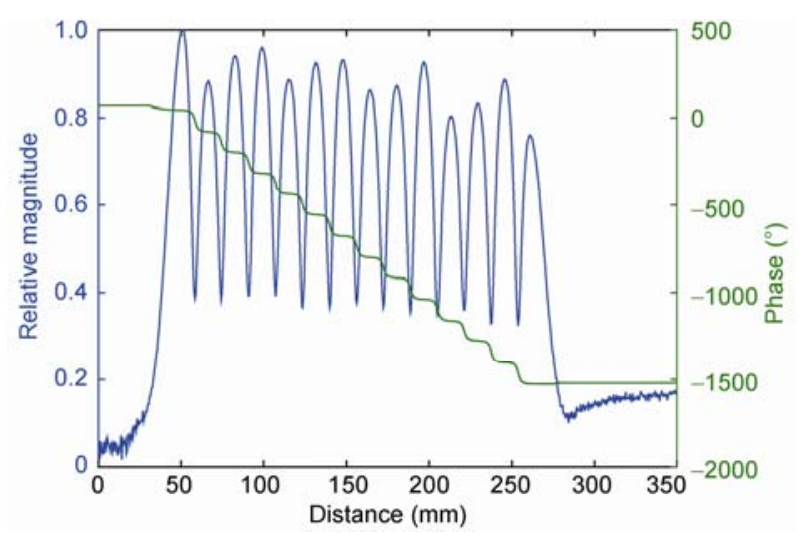

Figure 11 Field distribution after regular cells and output coupler tuning $(\mathrm{SWR}=1.1)$.

However, some perturbations result in slight differences. A higher electrical field for the left matching cell, which differs from that in Figure 6, is induced by a slight mismatch of the two couplers, and the noise level of the NWA is unstable, which results in an asymmetrical field distribution at the two ends in Figure 11.

\section{Conclusion}

A C-band traveling-wave accelerating structure was designed, and an experimental model with 12 regular cells and couplers was fabricated and tested. To a large extent, the RF cold test results agree well with the simulation, and therefore most crucial techniques of the design, fabrication and tuning method are verified systematically, particularly the design of the waveguide coupler, which is able to reduce the rate of RF breakdown.

The first phase of research and design of the structure has been accomplished, and it provides an integrated and systematic method for the research and design of a C-band accelerating structure. In the next step, the whole C-band accelerating structure will be fabricated and then high-power RF conditioning will be carried out.

We are grateful to Dr. Juwen Wang of the SLAC National Accelerator Laboratory for valuable suggestions and fruitful discussions. We would like to thank Professor Huaibi Chen, Qingxiu Jin and many other staff members at the Accelerator Laboratory of Tsinghua University and Suiming Zhou, Xing Sheng and Lifang Chen from China Electronics Technology Group Corporation for their machining and supports with microwave measurements. This work was supported by the Program of Shanghai Subject Chief Scientist-Natural Science Foundation (Key Program) (08XD14054).

1 Jiang M H, Yang X, Xu H J, et al. Shanghai Synchrotron Radiation Facility. Chinese Sci Bull, 2009, 54: 4171-4181

2 Kamitani T, Sugimura T, Yokoyama K, et al. Status of C-band accelerator module in the KEKB injector linac. In: Proceedings of PAC07, Albuquerque, New Mexico, USA, 2007, 2769-2771

3 Sakurai T, Inagaki T, Shirasawa K, et al. High power RF test on the C-band RF components of $8 \mathrm{GeV}$ accelerator for XFEL/SPring-8. In: Proceedings of PAC09, Vancouver, BC, Canada, 2009, 1563-1565

4 Helm R. A note on coupler asymmetries in long linear accelerator. Stanford Physics Reports, Menlo Park, CA, USA, 1960, M-167

5 Nantista C, Tantawi S, Dolgashev V, et al. Low-field accelerator structure couplers and design techniques. SLAC Pubs and Reports, Menlo Park, CA, USA, 2004, SLAC-PUB-10575

6 Yao C G. Electron Linear Accelerator (in Chinese). Beijing: Science Press, 1986. 143-150

7 Billen J, Young L. Poisson Superfish. Los Alamos National Laboratory Report, Santa Fe, New Mexico, USA, 2003, LA-UR-96-1834

8 Loew G, Miller R, Early R, et al. Computer calculation of traveling-wave periodic structure properties. IEEE Trans Nuclear Sci, 1979, NS-26: 3

9 Kroll N. Application of time domain simulation to coupler design for periodic structure. SLAC Pubs and Reports, Menlo Park, CA, USA, 2000, SLAC-PUB-8614

10 Steele C. A nonresonant perturbation theory. IEEE Trans Microwave Theory Tech, 1966, MTT-14: 2

11 Khabiboulline T, Dohlus M, Holtkamp N, et al. Tuning of a 50-cell constant gradient S-band travelling wave accelerating structure by using a nonresonant perturbation method. DESY Report, Hamburg, Germany, 1995, DESY M-95-02

Open Access This article is distributed under the terms of the Creative Commons Attribution License which permits any use, distribution, and reproduction in any medium, provided the original author(s) and source are credited. 